immerhin die Zusage abgetrotzt, bis Ende September Rezepte nicht zu retaxieren, auf denen Telefon und Arztvorname fehlen. Was im Umkehrschluss aber auch bedeutet, dass dies in absehbarer Zeit eben doch einen Anlass für Retaxationen geben könnte. Eine „endgültige Lösung“, so Splett, sei noch auszuhandeln.

Neben den Ersatzkassen und der AOK Rheinland-Hamburg wird auch die Knappschaft zunächst auf eine Rechnungskürzung verzichten, wenn Rezepte nach der Umstellung der Vorgaben zum 1. Juli nicht den neuesten Anforderungen entsprechen. Wie die Krankenkasse berichtet, wird sie eine dreimonatige Übergangsfrist gewähren, „da die Umstellung der Rezeptvordrucke noch nicht in allen Arztpraxen abgeschlossen ist".

Und wie verfährt der Apotheker nun mit Rezepten, die unvollständig ausgefüllt sind? Eigenhändig darf er laut AMVV nur ganz bestimmte Angaben auf dem Rezept ergänzen. Und das auch nur dann, „wenn ein dringender Fall vorliegt und eine Rücksprache mit der verschreibenden Person nicht möglich ist". Telefonnummer und Arztvorname gehören nicht dazu. Auch die etwa im Versorgungsvertrag mit den Ersatzkassen vorgesehene Möglichkeit, dass Apotheker fehlende Rezeptangaben selbst nachtragen, scheidet aus, solange der Vertrag nicht um eine entsprechende Erwähnung der neuen Formerfordernisse ergänzt wird.

Apotheker müssen Rezepte ohne Praxis-Telefonnummer und Arztvorname also zurück schicken. „Erhält der Arzt eine unvollständige Verordnung zurück, reicht es aus, wenn er die fehlenden Angaben handschriftlich ergänzt. Eine erneute Unterschrift ist nicht erforderlich", lautet jedenfalls auf Anfrage die Rechtsauffassung der ABDA. „Eine erneute Unterschrift wäre nur dann erforderlich, wenn die verordnete Leistung geändert würde."

Christoph Winnat

\title{
Patientendaten künftig direkt an MDK
}

Bis die Übermittlung der Patientendaten endlich elektronisch laufen kann, ist doch wenigstens einmal noch der bisherige Postweg aus Sicherheitsgründen zu modifizieren.

$\mathrm{K}^{\mathrm{r}}$ rankenkassen dürfen niedergelassene Ärzte nicht mehr auffordern, ihnen Unterlagen für den MDK in einem verschlossenen Umschlag zuzusenden. Sie müssen den Ärzten einen direkt an den MDK adressierten Rückumschlag zur Verfügung stellen. Zwar mussten Ärzte immer schon Unterlagen wie Krankenhausentlassberichte direkt an den MDK schicken, es war aber jahrelange Praxis, dass die Krankenkassen ihnen dafür einen Rückumschlag mit dem Vermerk „ärztliche Unterlagen nur vom MDK zu öffnen“ zur Verfügung gestellt hatten.

Das Problem: Viele Krankenkassen haben nach der Erfahrung der Bundesbeauftragten für den Datenschutz und die Informationsfreiheit (BfDI), Andrea Voßhoff, die Briefe widerrechtlich geöffnet. „Wie mir zudem bei Kontrollen aufgefallen ist, werden vom MDK in einem verschlossenen Umschlag erhaltene Unterlagen an die Krankenkasse zur dortigen Ablage offen zurückgegeben." Das bisher praktizierte Verfahren könne nicht mehr geduldet werden, sagte die Sprecherin der BfDI. „Für die Ärzte ändert sich nichts“, betonte sie. Der Ball liege weder bei ihnen noch bei den KVen, sondern ausschließlich bei den Krankenkassen.

Zurzeit laufen Gespräche zwischen dem GKV-Spitzenverband und der BfDI über die weitere Handhabung des Verfahrens. Offensichtlich ist die Erwartung, dass die Übermittlung der notwendigen Daten mittelfristig ohnehin elektronisch laufen wird. Die KBV weist darauf hin, dass von der Stellungnahme der BfDI das Gutachterverfahren nach der Psychotherapierichtlinie des G-BA nicht betroffen ist.

Ilse Schlingensiepen

\section{Ausbildung: MFA-Tarif nur begrenzt} unterschreitbar

Für die Gehälter von Auszubildenden zur MFA gelten strenge Regeln: Ausbilder hätten Azubis gemäß § 17 Abs. 1 Satz 1 des Berufsbildungsgesetzes eine „angemessene Vergütung" zu gewähren, so der Verband der medizinischen Fachberufe. Das Bundesarbeitsgericht habe mit einer aktuellen Entscheidung dargelegt (Az.: 9 AZR 108/14), dass eine Ausbildungsvergütung in der Regel dann nicht mehr angemessen ist, wenn sie die in einem einschlägigen Tarifvertrag geregelte Ausbildungsvergütung um mehr als $20 \%$ unterschreitet.

Rebekka Höhl

\section{Nur wenige Fortbildungsmuffel}

Die große Mehrheit der Vertragsärzte und -psychotherapeuten ist bei der Fortbildung am Ball. Laut KBV kommen $98 \%$ ihrer Fortbildungspflicht fristgerecht nach. Diese Erkenntnis zieht die KBV aus den Unterlagen, die Ärzte und Psychotherapeuten, die vor dem 30. Juni 2004 zugelassen waren, für den zweiten Nachweiszeitraum bis 30. Juni 2014 vorgelegt haben. Demnach waren 93.206 Ärzte und Psychotherapeuten aufgefordert, die verlangte Punktzahl zu dokumentieren. 91.410 davon hatten die 250 CMEPunkte fristgerecht nachgewiesen.

Thorsten Schaff

\section{KBV unterstützt Praxischefs in Sachen Hygiene}

Mit einem Fragebogen können niedergelassene Vertragsärzte den Hygienestatus in ihren Praxen überprüfen, wie die KBV mitteilt. Das Kompetenzzentrum Hygiene und Medizinprodukte der KBV habe den Fragebogen entwickelt, um Ärzten einen Überblick über die Rechtsvorschriften zu verschaffen und sie bei der Umsetzung zu unterstützen. Es gehe darum, ein Hygienemanagement zu etablieren, das den besten Infektionsschutz für Patienten wie für das Praxisteam gewährleiste.

Matthias Wallenfels

Der Fragebogen der KBV im Netz: http://goo.gl/EVXOiD

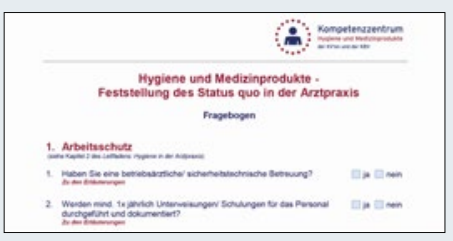

